\title{
Triage and Diagnosis of Chest Pain in Rural Hospitals: Implementation of the ACI-TIPI in the High Plains Research Network
}

\author{
Jobn M. Westfall, MD, MPH \\ Rebecca F. Van Vorst, MSPH \\ Joe McGloin, MS ${ }^{1}$ \\ Harry P. Selker, MD, MSPH \\ 'University of Colorado Health Sciences \\ Center, UCHSC at Fitzsimons, Aurora, Colo \\ ${ }^{2}$ Institute for Clinical Research and Health \\ Policy Studies, Tufts University School of \\ Medicine, Boston, Mass
}

Conflicts of interest: none reported

\section{CORRESPONDING AUTHOR}

John M. Westfall, MD, MPH

University of Colorado Health

Sciences Center

UCHSC at Fitzsimons

PO Box 6508, Mail Stop F496

Aurora, CO 80045-0508

jack.westfall@uchsc.edu

\begin{abstract}
PURPOSE The Acute Cardiac Ischemia Time-Insensitive Predictive Instrument (AClTIPI) has been shown to improve diagnostic accuracy of acute cardiac ischemia $(\mathrm{ACl})$ and decrease coronary care unit admissions in urban emergency departments. The purpose of this study was to determine the impact of the ACI-TIPI on triage and diagnosis of patients with chest pain in rural hospitals.
\end{abstract}

METHODS We undertook a controlled trial of the impact ACI-TIPI use in the High Plains Research Network (HPRN). Main outcome measures were the triage of patients in emergency departments (admission, transfer, or discharge home) and diagnostic accuracy.

RESULTS There were 1,861 patients seen during a 10-month period. Forty-five percent of all patients complaining of chest pain were discharged home from the emergency department. Eight percent were transferred from the emergency department, and another 10\% were transferred later after admission. Among patients with acute myocardial infarction or unstable angina, $22.2 \%$ were transferred directly from the emergency department and only $3 \%$ were discharged home when ACI-TIPI was available, compared with $18.7 \%$ transferred and 5.2\% discharged home when not available $(P=.4)$. Diagnostic accuracy was high and not statistically different with the addition of the ACI-TIPI score $(86.8 \% \mathrm{ACI}-\mathrm{TIPI}$ off vs $89.0 \% \mathrm{ACl}$-TIPI on, $P=.15)$,

CONCLUSIONS Physicians in the HPRN provided appropriate diagnosis and triage to patients with chest pain. Routine addition of the ACI-TIPI score did not improve diagnostic accuracy or significantly change triage. Further research on ACI-TIPI in rural hospitals is necessary before recommending routine use of the ACI-TIPI.

Ann Fam Med 2006:4:153-158. DOI: 10.1370/afm.403.

\section{INTRODUCTION}

T schemic heart disease resulting in acute myocardial infarction leads to more than 1 million hospitalizations every year in the United States.

Lack of clear diagnosis has been reported as one reason for inadequate use of proven treatments. ${ }^{1}$ The Acute Cardiac Ischemia Time-Insensitive Predictive Instrument (ACI-TIPI) assists in the diagnosis of acute cardiac ischemia by printing the probability ( $0 \%$ to $100 \%)$ on the electrocardiogram header that the patient is truly suffering acute cardiac ischemia). The ACI-TIPI has been shown to improve the diagnostic accuracy and the triage of patients with chest pain in urban and suburban emergency departments. Specifically, the ACI-TIPI has been shown to decrease unnecessary admissions to the hospital and coronary care unit among patients without true acute ischemia heart disease. ${ }^{2}$

Because many rural and frontier hospitals have limited resources or no coronary care unit, patients requiring a coronary care unit admission are often transferred to another hospital. Unnecessary transfer from a rural 
hospital to an urban hospital is disruptive and stressful for patients and their family and generates enormous costs. One study found that $30 \%$ to $50 \%$ of patients with acute cardiac ischemia are transferred at some point during their care. ${ }^{3}$ No data exist on the incidence of unnecessary interhospital transfer.

The purpose of this study was to evaluate the impact of the use of ACI-TIPI on triage decisions and diagnostic accuracy for patients coming to rural and frontier emergency departments with chest pain or other symptoms suggestive of acute cardiac ischemia. We hypothesized that the use of the ACI-TIPI score might decrease the rate of interhospital transfer for patients without acute ischemia while not affecting the admission and/or transfer decisions for patients truly suffering acute ischemia.

\section{METHODS}

\section{Study Setting}

Our study, a controlled trial, was conducted in the High Plains Research Network (HPRN) in rural northeast Colorado. The HPRN is an integrated practicebased research network consisting of the hospitals, emergency departments, ambulatory clinical practices, and clinicians in the northeast region of Colorado. The HPRN is largely rural and frontier, includes nearly 15,000 square miles, and has a population of approximately 90,000. At the time of the study, the HPRN included approximately 60 family physicians, 5 internists, 12 nurse practitioners, and 15 physician's assistants. Ten hospitals participating in this study varied in size from 6 to 40 beds. We included 1 ambulatory clinic with emergency capabilities. Two hospitals had limited intensive care unit capabilities; however, there were no cardiologists whose practice was located in these regions, and no hospital had heart catheterization capabilities. All the hospitals provided thrombolytic therapy to appropriate patients. In the HPRN, 65\% of patients with an acute myocardial infarction are transferred to an urban hospital. ${ }^{3}$ The closest tertiary care center was 50 to 180 miles away.

\section{Intervention}

We obtained 16 electrocardiogram (ECG) machines on loan for the duration of the study from Agilent Technologies (Palo Alto, Calif; formerly Hewlett-Packard) and GE/Marquette of GE Healthcare (Waukesha, Wisc), the only electrocardiographic equipment with the ACI-TIPI software then available. Neither Agilent Technologies nor GE Healthcare had any input into research design, data analysis, reporting, or publication.

The ACI-TIPI reporting mechanism can be turned on or off. When on, the ACI-TIPI software provides the printed probability report along with the standard ECG interpretation report. When off, only the standard ECG report is printed.

We held educational sessions in each community during the first 3 months to teach physicians, nurses, and emergency department staff how to operate the new electrocardiographic equipment and provided a notebook with educational materials and study protocols to each clinician and hospital. The trial was conducted during the next 10 months. According to the design of the ACI-TIPI trial, each month the hospitals alternated between control (ACI-TIPI off) and intervention (ACI-TIPI on). ${ }^{2}$ Every ECG performed in the intervention months was intended to include the ACI-TIPI report along with the standard ECG report. Every ECG performed in the control months included only the standard ECG report. We randomly assigned hospitals to begin with the ACI-TIPI on or off. When finished, each site provided intervention data for 5 months and control data for 5 months.

\section{Data Collected and Analysis}

The following ACI-TIPI data were acquired when the patient had the ECG: patient age and sex; whether the patient had chest pain or left arm pain, and whether this pain was the primary complaint ${ }_{i}$ and the electrocardiographic computer analysis of the ECG waveforms. The ACI-TIPI then calculated the probability score.

A list of patients was generated from the electrocardiograph memory log. Initial review was done on all patients who received an ECG. Approximately 70\% of these ECGs were done for reasons other than acute chest pain, eg, preoperative evaluation, follow-up evaluation for chronic heart disease, and so on. Thorough medical record review was undertaken only for those patients with symptoms suggestive of acute cardiac ischemia (eg, chest pain, pressure, tightness, radiating pain to the neck or jaw, unexplained shortness of breath, epigastric pain, diaphoresis, weakness, or dizziness).

Data obtained included demographic variables, patient complaints, ECG, treatments, disposition, and the physician's final diagnosis using standard ICD9-CM? diagnostic codes. A trained medical record abstractor performed all record reviews at each hospital. Each patient case was reviewed, and diagnoses of acute cardiac ischemia were confirmed on the basis of signs and symptoms, clinical course, ECGs, and serum cardiac biomarker tests. ${ }^{4}$ We used a modified version of the World Health Organization criteria to confirm acute myocardial infarction. ${ }^{4}$ Because many patients were transferred from the emergency department, we did not always have serial measurements of cardiac biomarkers and ECGs. We considered any elevated serum creatine kinase- $\mathrm{MB}$ fraction or troponin level 
as a positive serum marker. An ECG with a report of ischemia or infarction was considered a positive ECG A patient needed to have 2 of the 3 following indicators for diagnosis of an acute myocardial infarction: clinical symptoms suggestive of acute cardiac ischemia, elevated serum cardiac biomarkers, and ECG showing ischemia or infarction. For unstable angina a patient required clinical symptoms suggestive of acute cardiac ischemia and a final diagnosis of unstable angina by the attending physician. This study was approved by the Combined Multiple Institution Review Board at the University of Colorado Health Sciences Center.

We compared the rates of discharge, admission, and transfer between patients evaluated during intervention and control months. First, we dichotomized the triage into home from emergency department vs all other triage decisions (admit, transfer, transfer after admit). Second, we dichotomized the disposition into any transfer (transfer from emergency department and transfer after admit) vs not transferred. Based on work by Selker et $\mathrm{al}_{1}^{2}$ we defined the following diagnostic groups and recommended triage decisions: (1) patients with acute cardiac ischemia (acute myocardial infarction or unstable angina) should be admitted or transferred ${ }_{i}(2)$ patients with stable angina could be discharged home; and (3) patients without cardiac ischemia could be discharged home. Comparison of patient characteristics between the control and intervention periods used $t$ tests for continuous variables and $\chi^{2}$ tests for categorical variables.

\section{Table 1. Demographics and Diagnosis of Patients With Acute Cardiac Symptoms}

\begin{tabular}{|c|c|c|c|}
\hline $\begin{array}{l}\text { Demographic } \\
\text { Characteristics }\end{array}$ & $\begin{array}{c}2001-2002 \\
\text { Control } \\
\text { ACI-TIPI Off } \\
(n=994)\end{array}$ & $\begin{array}{l}2001-2002 \\
\text { Intervention } \\
\text { ACI-TIPI On } \\
(n=867)\end{array}$ & $P$ Value \\
\hline Sex, \% & & & .3 \\
\hline Female & 51.1 & 53.4 & \\
\hline Male & 48.9 & 46.6 & \\
\hline Age, mean y & 62.3 & 61.4 & .7 \\
\hline Race, \% & & & .4 \\
\hline White & 81.8 & 81.5 & \\
\hline Black & 0.3 & 0.1 & \\
\hline Hispanic & 9.7 & 10.8 & \\
\hline Other or unable to determine & 9.2 & 7.6 & \\
\hline Final diagnosis, \% & & & .1 \\
\hline $\begin{array}{l}\text { Acute myocardial infarction } \\
\text { (ICD-9-CM 410) }\end{array}$ & 7.8 & 5.8 & \\
\hline $\begin{array}{l}\text { Acute cardiac ischemia } \\
\quad(\text { ICD-9-CM 410, 411) }\end{array}$ & 13.8 & 11.7 & \\
\hline Stable angina (ICD-9-CM 413) & 3.4 & 2.0 & \\
\hline Noncardiac or not acute ischemia & 82.8 & 86.3 & \\
\hline ACI-TIPI score, mean & 28.0 & 24.6 & .5 \\
\hline
\end{tabular}

We were interested in both the appropriateness of triage decisions among all patients and the accuracy of diagnosing an acute myocardial infarction. Accuracy was simply defined as the number of patients with a correct diagnosis or correct triage divided by the total sample. A formal description can be found in the Supplemental Appendix, found online only at http://www. annfammed.org/cgi/content/full/4/2/153/DC1. We used a Mantel-Haenszel test to compare diagnostic and triage accuracy between ACI-TIPI on and off months controlling for site of care. With 250 patients in the intervention and control groups, this study has a power of $88 \%$ to detect an absolute difference in discharge rate of $10 \%(\alpha=.1,1$-tailed test, change in discharge home rate from $25 \%$ to $35 \%$ ).

\section{RESULTS}

Table 1 displays the patient demographics and compares them between months when ACI-TIPI was on and months when ACI-TIPI was off. No differences in patient demographics were found. Eighty-one percent had chest pain as their chief complaint. Nearly $97 \%$ of all patients had serum cardiac markers obtained in the emergency department. Greater than $99 \%$ of patients had an ECG in the emergency department. Overall, $45.4 \%$ of patients in this study were discharged home from the emergency department, $8 \%$ were transferred directly from the emergency department, 10\% were admitted to the rural hospital then transferred in the subsequent days, and $36 \%$ were admitted to and cared for exclusively in the rural hospital.

Table 2 displays the triage and disposition for patients with various diagnoses. In patients with a final diagnosis of no acute cardiac ischemia, there was no significant change in the rate of discharge home from emergency department when ACITIPI was available. For patients with acute cardiac ischemia (ICD-9-CM $410,411)$ there was a small, nonsignificant decrease in the percentage of patients inappropriately discharged home (5.2\% ACI-TIPI off vs 3.0\% ACI-TIPI on, $P=.4$ ). Patients with acute ischemia were appropriately hospitalized $97 \%$ of the time. By far, the largest group of patients was the group who complained of chest pain but were not having acute ischemia. Nearly one half of these patients were admitted to the hospital. Most 
patients with an acute myocardial infarction were transferred to a tertiary care hospital, and there was no difference in overall transfer rate associated with the availability of the ACI-TIPI score.

In 8 of the 11 facilities the diagnostic accuracy for acute myocardial infarction improved during months when the ACI-TIPI was used (Table 4). For all hospitals, however, the average diagnostic accuracy was not statistically different (86.8\% ACI-TIPI off vs $89.0 \%$ ACI-TIPI on, $P=.15)$. The true-positive rate remained essentially the same (3.9\% ACI-TIPI off, $4.0 \%$ ACI-TIPI on). The true-negative rate increased from $82.9 \%$ to $85.0 \%$. As expected from previous research, the false-positive rate decreased from $13.2 \%$ to $9.7 \%$. Unfortunately, the false-negative rate increased from $0 \%$ to $1.3 \%$. Only 3 $(2.3 \%)$ patients with an ICD-9-CM diagnosis of acute myocardial infarction (410) were discharged from the emergency department. One additional patient that met clinical criteria for an acute myocardial infarction was discharged home. Because there were only a few cases at each hospital, no change reached statistical significance.

\section{DISCUSSION}

The purpose of this study was to determine the impact of providing the ACI-TIPI score on triage and diagnostic accuracy for patients complaining of chest pain to rural and frontier hospitals. We found that the ACITIPI score had no statistically significant impact on triage or diagnostic accuracy. The small changes in triage and diagnostic accuracy we noted were consistently in the same direction of improving clinical care. Because of the small sample size for each diagnostic category, however, these changes did not reach statistical significance. Overall, diagnostic and triage accuracy were at or above that reported in previous studies. ${ }^{2,5}$

Unfortunately, there was no change in the triage among patients without acute ischemia. Although many patients with chest pain have other diagnoses that require hospitalization (pneumonia, heart failure, upper gastrointestinal bleeding), only a small proportion of patients without acute ischemia were transferred (11\%), roughly equivalent to the rate of admission to the coronary care unit among patients

Table 2. Triage and Disposition of Patients With Acute Cardiac Symptoms

\begin{tabular}{|c|c|c|c|c|}
\hline Triage and Disposition Characteristics & $\begin{array}{l}2001-2002 \\
\text { Control } \\
\text { ACI-TIPI Off }\end{array}$ & $\begin{array}{l}2001-2002 \\
\text { Intervention } \\
\text { ACI-TIPI On }\end{array}$ & $\begin{array}{c}\text { Home From } \\
\text { Emergency } \\
\text { Department } \\
\text { vs All Others } \\
P \text { Value }\end{array}$ & $\begin{array}{c}\text { Any Transfer } \\
\text { vs No Transfer } \\
P \text { Value }\end{array}$ \\
\hline No. of patients without acute cardiac ischemia & 807 & 730 & .4 & .2 \\
\hline Transfer from emergency department, \% & 5.6 & 6.8 & & \\
\hline Transfer after admit, \% & 5.1 & 6.1 & & \\
\hline Admit, no transfer, \% & 36.1 & 35.9 & & \\
\hline Home from emergency department, no transfer, \% & 53.2 & 51.2 & & \\
\hline $\begin{array}{l}\text { No. of patients with myocardial infarction } \\
\text { (ICD-9-CM 410) }\end{array}$ & 78 & 50 & .8 & .7 \\
\hline Transfer from emergency department, \% & 19.2 & 24.0 & & \\
\hline Transfer after admit, \% & 53.8 & 46.0 & & \\
\hline Admit, no transfer, \% & 24.4 & 28.0 & & \\
\hline Home from emergency department, no transfer, \% & 2.6 & 2.0 & & \\
\hline No. of patients with unstable angina (ICD-9-CM 411) & 56 & 49 & .3 & .3 \\
\hline Transfer from emergency department, \% & 17.9 & 20.4 & & \\
\hline Transfer after admit, \% & 37.5 & 24.5 & & \\
\hline Admit, no transfer, \% & 35.7 & 51.0 & & \\
\hline Home from emergency department, no transfer, \% & 8.9 & 4.1 & & \\
\hline $\begin{array}{l}\text { No. of patients with acute cardiac ischemia } \\
\text { (ICD-9-CM 410,411) }\end{array}$ & 134 & 99 & .4 & .2 \\
\hline Transfer from emergency department, \% & 18.7 & 22.2 & & \\
\hline Transfer after admit, \% & 47.0 & 35.4 & & \\
\hline Admit, no transfer, \% & 29.1 & 39.4 & & \\
\hline Home from emergency department, no transfer, \% & 5.2 & 3.0 & & \\
\hline No. of patients with stable angina (ICD-9-CM 413) & 33 & 17 & .2 & .4 \\
\hline Transfer from emergency department, \% & 3.0 & 0.0 & & \\
\hline Transfer after admit, \% & 18.2 & 11.8 & & \\
\hline Admit, no transfer, $\%$ & 54.6 & 47.0 & & \\
\hline Home from emergency department, no transfer, \% & 24.2 & 41.2 & & \\
\hline
\end{tabular}




\begin{tabular}{|c|c|c|c|c|}
\hline Condition & $\begin{array}{l}2001-2002 \\
\text { Control } \\
\text { ACI-TIPI Off } \\
(n=974)\end{array}$ & $\begin{array}{l}2001-2002 \\
\text { Intervention } \\
\text { ACI-TIPI On } \\
(n=846)\end{array}$ & $\begin{array}{c}\% \\
\text { Change }\end{array}$ & $\begin{array}{c}P \\
\text { Value }\end{array}$ \\
\hline $\begin{array}{l}\text { Acute myocardial infarction } \\
\text { (ICD-9-CM 410) }\end{array}$ & 97.4 & 98.0 & +0.6 & .8 \\
\hline $\begin{array}{l}\text { Unstable angina } \\
\text { (ICD-9-CM 411) }\end{array}$ & 91.1 & 95.9 & +4.8 & .3 \\
\hline $\begin{array}{l}\text { Acute cardiac ischemia } \\
\text { (ICD-9-CM 410, 411) }\end{array}$ & 94.7 & 97.0 & +2.3 & .6 \\
\hline Stable angina (ICD-9-CM 413) & 24.2 & 41.2 & +17 & .5 \\
\hline Not acute cardiac ischemia & 52.1 & 50.8 & -1.3 & 6 \\
\hline \multicolumn{5}{|c|}{$\begin{array}{l}\text { ACI-TIPI = Acute Cardiac Ischemia Time-Insensitive Predictive Instrument. ICD-9-CM = International Classification } \\
\text { of Disease, 9th Edition, Clinical Modification. }\end{array}$} \\
\hline \multicolumn{5}{|c|}{$\begin{array}{l}\text { Note: Based on work by Selker et } \mathrm{al}^{2} \text { we defined the following diagnostic groups and recommended triage deci- } \\
\text { sions: (1) patients with acute cardiac ischemia (acute MI or unstable angina) should be admitted or transferred, } \\
\text { (2) patients with stable angina could be discharged home, and (3) patients without cardiac ischemia could be } \\
\text { discharged home. }\end{array}$} \\
\hline
\end{tabular}

In a rural hospital without advanced cardiac services, transfer may be viewed as a treatment option, as is the use of aspirin, $\beta$-blockers, and thrombolytics. Although there are evidencebased guidelines for the medical treatments of acute myocardial infarction, there are no guidelines aiding the decision of whether to transfer a patient suffering from acute cardiac ischemia. Transfer appears to be a complex decision and was not dramatically altered by the additional diagnostic information obtained using the ACI-TIPI program. Further research on the relationship
Table 4. Rate of Diagnostic Accuracy for Acute Myocardial Infarction With and Without the Use of ACI-TIPI

\begin{tabular}{lccc}
\hline & $\begin{array}{c}\text { 2001-2002 } \\
\text { Control } \\
\text { ACl-TIPI Off } \\
\text { (n = 974) }\end{array}$ & $\begin{array}{c}\text { 2001-2002 } \\
\text { Intervention } \\
\text { ACl-TIPI On } \\
\text { (n = 846) }\end{array}$ & \% Change \\
\hline Hospital 1 & 88.8 & 90.7 & +1.9 \\
Hospital 2 & 80.0 & 87.5 & +7.5 \\
Hospital 3 & 85.7 & 88.5 & +2.8 \\
Hospital 4 & 92.2 & 90.0 & -2.2 \\
Hospital 5 & 85.5 & 87.7 & +2.2 \\
Hospital 6 & 91.6 & 93.6 & +2.0 \\
Hospital 7 & 93.9 & 95.2 & +1.3 \\
Hospital 8 & 70.0 & 100.0 & +30.0 \\
Hospital 9 & 82.6 & 87.1 & +4.5 \\
Hospital 10 & 82.8 & 79.4 & -3.4 \\
Hospital 11 & 85.1 & 83.7 & -1.4 \\
All hospitals* & 86.8 & 89.0 & +2.2 \\
\hline ACl-TIPI = Acute Cardiac Ischemia Time-Insensitive Predictive Instrument. \\
* P = .15.
\end{tabular}

without acute ischemia in ACI-TIPI research in urban hospitals ( $7 \%$ to $15 \%)^{2}$

The patients in our study were similar in age, sex, and chief complaint to patients in previous studies on ACI-TIPI. ${ }^{2}$ Nearly all patients had ECG and serum cardiac enzyme testing implying that these patients were indeed considered by the treating physician as potentially having acute ischemic heart disease. Only 4 patients who met clinical criteria for acute myocardial infarction were discharged home from the emergency department, a rate similar to that found in previous research. ${ }^{2}$ Unfortunately, we have no follow-up data on this group of patients to determine final diagnosis and outcome. of hospital, physician, and patients factors related to transfer may help identify patient groups most likely to benefit from transfer.

There were several limitations to this study. First, the newest version of the ACI-TIPI requires that the ECG machine print 2 complete 12 -lead ECG reports, one with a traditional interpretation and a second with the ACI-TIPI score. We found several occasions in which the physician was not seeing the ECG report with the ACI-TIPI score. The ACI-TIPI score could be bypassed by a manual override, and we heard of several occasions where this was done and no score was generated. We suggest that the ACI-TIPI score be part of the standard ECG printout. Second, we have no way of knowing when the ACI-TIPI score was actually used by the physician to help with the diagnosis and triage. Comments about the ACI-TIPI score were not a routine part of the patient record.

Third, because we had so few patients with any specific diagnosis, the changes we found in diagnostic accuracy and triage did not reach statistical significance. We anticipated more patients with each diagnosis and a larger effect size. Our rate of acute cardiac ischemia (13\%) was lower than previously reported $(17.5 \%){ }^{2}$ For patients without acute ischemia $(\mathrm{n}=$ 1,537 ), we had $90 \%$ power to detect a $7.5 \%$ absolute change in triage, but we found only a $2 \%$ to $3 \%$ change in triage in this group. For patients with acute ischemia ( $n=233$ ) we needed a $20 \%$ absolute change in triage to have $90 \%$ power to detect a significant change, but found only a $2 \%$ to $8 \%$ change in triage. Given the total sample, we had $90 \%$ power to detect a $4.4 \%$ change in diagnostic accuracy. A larger study or one with prospective data collection and determination on the actual use of the ACI-TIPI score is needed to determine whether ACI-TIPI improves outcomes 
in rural hospitals. Finally, because we did not abstract the charts at urban hospitals where patients were transferred, we were unable to confirm acute myocardial infarction in some patients. There may have been patients transferred with a diagnosis of acute myocardial infarction who had a different final diagnosis from the urban hospital. From a previous study we found that $83 \%$ of patients transferred with a diagnosis of acute myocardial infarction had a final diagnosis of acute myocardial infarction or unstable angina. ${ }^{3}$

The diagnostic accuracy of acute myocardial infarction has been a concern in previous research. ${ }^{6-8} \mathrm{We}$ found that because so many patients who appear to have acute myocardial infarction get transferred, the rural hospital data are often insufficient to meet the standard WHO criteria for a formal diagnosis of acute myocardial infarction. We chose a conservative measure for the diagnosis of acute myocardial infarction and found excellent overall agreement between the ICD-9CM diagnosis of acute myocardial infarction and the clinical diagnosis of acute myocardial infarction. Most disagreement between ICD-9-CM diagnosis and clinical diagnosis was an error in overdiagnosing acute ischemia or infarction even when the patient did not meet clinical criteria. Defining an acute myocardial infarction among transferred patients will continue to be a research dilemma and deserves further investigation.

The benefit of this study is that it was a real-life implementation and effectiveness trial of the ACI-TIPI in rural hospitals. It included only the type and amount of education that would occur in a typical nonresearch setting, which may not be adequate to fully educate physicians about the interpretation and benefit of the use of the ACI-TIPI score. This type of research points to the value of a practice-based research network that can collect and aggregate data on important medical conditions for rural primary care physicians.

Physicians in the HPRN diagnosed acute cardiac ischemia and triaged patients accurately. The use of the ACI-TIPI did not appear to have a significant impact on the triage and diagnosis for patients with chest pain. Inclusion of the ACI-TIPI score into the standard ECG interpretation report may make it easier to use. Conducting this type of study in more rural sites may provide a sample sufficient to detect statistically significant changes in triage and diagnostic accuracy.

To read or post commentaries in response to this article, see it online at http://www.annfammed.org/cgi/content/full/4/2/153.
Key words: Chest pain; myocardial infarction; myocardial ischemia; practice-based research; electrocardiography; blood circulation

Submitted April 19, 2005; submitted, revised, August 23, 2005; accepted August 25, 2005.

A version of this report was presented as a distinguished paper at the Annual Meeting of the North American Primary Care Research Group in Banff, Canada, October, 2003.

Funding support: Funded by the Agency for Health Care Research and Quality grant No. R03 HS1100301. Agilent Technologies and GE/Marquette loaned the electrocardiogram machines to the hospitals for the duration of the study. The Caring for Colorado Foundation provided funds to purchase all the electrocardiogram machines for the hospitals. Neither the funding agencies nor the commercial vendors had input into the research design, data analysis, reporting, or publication.

Acknowledgments: We are grateful to Manlik Kwong and Joni Beshansky, RN, MPH, for their assistance in the conception and implementation of this project.

Study participants: The High Plains Research Network hospitals participating in this study: Lincoln Community Hospital, Hugo; Plains Medical Center, Limon; Melissa Memorial Hospital, Holyoke; East Morgan County Hospital, Brush; Keefe Memorial Hospital, Cheyenne Wells; Colorado Plains Medical Center, Fort Morgan; Haxtun Hospital District, Haxtun; Wray Community District Hospital, Wray; Yuma District Hospital, Yuma; Sterling Regional MedCenter, Sterling; and Sedgwick County Memorial Hospital, Julesburg.

\section{References}

1. Selker HP, Griffith JL, Beshansky JR, et al. Patient-specific predictions of outcomes in myocardial infarction for real-time emergency use: a thrombolytic predictive instrument. Ann Intern Med. 1997;127:538-556.

2. Selker HP, Beshansky JR, Griffith JL, et al. Use of the acute cardiac ischemia time-insensitive predictive instrument (ACI-TIPI) to assist with triage of patients with chest pain or other symptoms suggestive of acute cardiac ischemia. A multicenter, controlled clinical trial. Ann Intern Med. 1998;129:845-855.

3. Westfall JM, McGloin J. Impact of double counting and transfer bias on estimated rates and outcomes of acute myocardial infarction. Med Care. 2001;39:459-468.

4. Gillum RF, Fortmann SP, Prineas RJ, Kottke TE. International diagnostic criteria for acute myocardial infarction and acute stroke. Am Heart J. 1984;108:150-158.

5. McCarthy BD, Wong JB, Selker HP. Detecting acute cardiac ischemia in the emergency department: a review of the literature. J Gen Intern Med. 1990;5:365-373.

6. Green J, Wintfeld N. How accurate are hospital discharge data for evaluating effectiveness of care? Med Care. 1993;31:719-731.

7. lezzoni LI, Burnside S, Sickles L, et al. Coding of acute myocardial infarction. Clinical and policy implications. Ann Intern Med. 1988; 109:745-751.

8. Austin PC, Daly PA, Tu JV. A multicenter study of the coding accuracy of hospital discharge administrative data for patients admitted to cardiac care units in Ontario. Am Heart J. 2002;144:290-296. 